ELSEVIER

\title{
Structural insights into the central complement component C3
}

\author{
Bert J.C. Janssen, Piet Gros* \\ Crystal and Structural Chemistry, Bijvoet Center for Biomolecular Research, Department of Chemistry, Faculty of Science, \\ Utrecht University, Padualaan 8, 3584 CH Utrecht, The Netherlands
}

Received 2 June 2006; accepted 22 June 2006

\begin{abstract}
$\mathrm{C} 3$ is a central protein of the complement system, which is important to immune defense and provides a link between innate and adaptive immunity. Three pathways of complement activation converge at the activation of $\mathrm{C} 3$ yielding a diverse set of biological responses. This versatile and flexible molecule interacts with various proteins to fulfill its functions. Here we review recent insights gained from the crystal structure determinations of human, native $\mathrm{C} 3$ and its physiological down-regulation product $\mathrm{C} 3 \mathrm{c}$. The data provided, for the first time, a complete and detailed view of the composition and arrangement of the domains in C3. Comparison of C3 with C3c indicates marked flexibility of the molecule, particularly in the $\alpha$-chain. We discuss the observed domain rearrangements, conformational changes and the location of various protein binding sites. These detailed, and structural, insights are important for developing models of the molecular mechanisms underlying the diverse biological activities of this large and complex molecule.
\end{abstract}

(C) 2006 Published by Elsevier Ltd.

Keywords: Complement system; C3; $\alpha 2$-macroglobulin family; Innate immunity; Structure

\section{Introduction}

The mammalian complement system plays an important role in humoral immune defense against invading microorganisms [reviewed in (Carroll, 2004; Walport, 2001)]. The system may recognize and eliminate pathogens in the blood stream, elicit inflammatory responses and facilitate the adaptive immune response. Besides recognition and clearance of "non-self" material, the complement system is involved in clearance of "self" particles, such as apoptotic cells. Unduly complement activity, however, has been associated with a wide variety of inflammatory conditions and immune-complex diseases such as autoimmune diseases, sepsis, adult respiratory distress syndrome, hyperacute rejection of transplants, etc. In this sense, the complement system has often been referred to as a double-edged sword.

Complement activity is established by the interplay of 30-40 soluble plasma proteins and cell-surface proteins. Three routes of recognition lead to activation of the complement system: the classical (antibody-mediated) pathway, the lectin pathway and the alternative pathway. These pathways converge in the

\footnotetext{
* Corresponding author. Tel.: +31 30253 3502; fax: +31 302533940 .

E-mail address: p.gros@chem.uu.nl (P. Gros).
}

activation of the third complement component $\mathrm{C} 3 . \mathrm{C} 3$ is activated proteolytically by enzyme complexes, the $\mathrm{C} 3$ convertases, yielding the anaphylatoxin $\mathrm{C} 3 \mathrm{a}$, which mediates inflammation (Bokisch et al., 1969), and the major fragment C3b. C3b can covalently attach to target surfaces via its reactive thioester (Law et al., 1979). Bound C3b provides a platform for the generation of more $\mathrm{C} 3$ convertases yielding amplification of the complement response (Muller-Eberhard and Gotze, 1972) and formation of C5 convertases initiating the terminal phase of complement activation, i.e. formation of the membrane-attack complex on target cells resulting in cell lysis. Furthermore, C3b may opsonize particles leading to phagocytosis and, thus, clearance of the tagged particles. Subsequent proteolysis of $\mathrm{C} 3 \mathrm{~b}$ results in the formation of fragment $\mathrm{iC} 3 \mathrm{~b}$, and finally fragments $\mathrm{C} 3 \mathrm{dg}$ and C3c (Harrison and Lachmann, 1980; Ross et al., 1982). iC3b and C3dg may stimulate B-cells providing a link between the innate and adaptive immune responses (Carroll, 2004). To fulfill all these roles, a diverse set of protein molecules interact with the various activated fragments of $\mathrm{C} 3$. This includes the protease of the (alternative pathway) convertases, complement regulators and cell-surface receptors on macrophages and Bcells (Lambris, 1988). The specificity of binding partners implies the existence of cryptic binding sites and, therefore, the importance of conformational differences between the $\mathrm{C} 3$ fragments. 
$\mathrm{C} 3$ and the homologous complement proteins $\mathrm{C} 4$ and $\mathrm{C} 5$ are members of the $\alpha 2$-macroglobulin $(\alpha 2 \mathrm{M})$ family of proteins. The proteins of this family have emerged over 700 million years ago (Sunyer et al., 1998). They are ubiquitous among metazoans and have a primary function in host defense (Blandin and Levashina, 2004). Other well known members of the family include the universal protease inhibitor $\alpha 2$-macroglobulin and the thioester-containing proteins (TEPs) from insects and nematodes (Budd et al., 2004). All these proteins are relatively large (1400-1800 amino-acid residues) with several marked functional features; a reactive thioester moiety enabling covalent attachment to target particles, a highly variable central segment likely involved in recognition; and, the propensity to undergo conformational changes yielding distinct protein-binding interactions (Budd et al., 2004; Chu and Pizzo, 1994; Lagueux et al., 2000). Structural insights into these large proteins, however, have remained scarce and fragmented.

Here, we focus on recent insights that have been obtained through the structure determination of the full-length, native $\mathrm{C} 3$ molecule with its thioester moiety intact and the $\mathrm{C} 3 \mathrm{c}$ molecule, which is the major breakdown product of the C3 molecule (Janssen et al., 2005). These structures provided for the first time insights into the intricate domain arrangement, the thioester protection mechanisms and flexibility of this type of proteins. With these structures available we can now develop detailed models for the underlying molecular mechanisms of C3 activation and regulation.

\section{Structure determination of $\mathbf{C 3}$}

The three-dimensional structure of C3, or of any of its homologues, has remained elusive for a long period of time. Typically for molecules of this size, the approach of structural biologists has been to determine structures of fragments or domains first. In the 1980s the first crystal structure determined of a fragment from a member of the $\alpha 2 \mathrm{M}$ family was that of the anaphylatoxin C3a (Huber et al., 1980), followed 9 years later by the NMR solution structure of the homologous C5a (Zuiderweg et al., 1989). Since then only three other domain structures were determined by crystallography or NMR: (i) in 1998 the receptor-binding domain (RBD) of $\alpha 2 \mathrm{M}$ (Jenner et al., 1998); (ii) the structure of C3d in 1998 (Nagar et al., 1998) and more recently (in 2002) that of the homologous C4d (van den Elsen et al., 2002); and finally (iii) most recently, in 2005, the C-terminal domain C345C of C5 (Bramham et al., 2005). These data provided structural insights for $40 \%$ of the protein molecule. However, the structure of the remaining $60 \%$ and the overall architecture remained elusive. This is exemplified by a conserved-domain search at http://www.ncbi.nlm.nih.gov/Structure/cdd/wrpsb.cgi. This site presents two large single domain regions for $\mathrm{C} 3$ : A2M_N (pfam01835) the $\alpha 2 \mathrm{M}$ family $\mathrm{N}$-terminal region corresponding to the $\beta$-chain of $\mathrm{C} 3$; and, $\mathrm{A} 2 \mathrm{M}$ (pfam00207) the $\alpha 2 \mathrm{M}$ family $\mathrm{C}$-terminal region constituting most of the $\alpha$-chain.

In our structural studies on C3 (Janssen et al., 2005) we first crystallized the proteolytic fragment $\mathrm{C} 3 \mathrm{c}$, which constitutes $72 \%$ of the complete $\mathrm{C} 3$. Analogous to limited proteolysis treatment, the naturally occurring breakdown of $\mathrm{C} 3$ into $\mathrm{C} 3 \mathrm{c}$ in blood plasma yields a molecule forming the core structure of the molecule. Generally, such a core molecule exhibits less flexibility and is more amenable to crystallization. In addition, the molecule was further trimmed by enzymatic deglycosylation completely removing the glycan moiety on Asn-917. Crystals of this deglycosylated $\mathrm{C} 3 \mathrm{c}$ typically diffracted to $\sim 3-\AA$ resolution with the best crystal diffracting to $2.4-\AA$ resolution. Crystalstructure determination was based on the classical heavy-atom derivative method. More expeditious methods were not applicable, because no significant structural models were available $a$ priori for $\mathrm{C} 3 \mathrm{c}$ to be used in molecular replacement; and, SeMetMAD phasing was excluded because the protein was obtained from the natural, human source. In the end, the structure was determined by phasing from two heavy-atom derivatives and phase combination using 6-fold averaging over three crystals. The final electron-density map was of excellent quality. The final model of $\mathrm{C} 3 \mathrm{c}$ consisted of 1109 residues forming 10 domains (coordinates of this model are available; entry 2A74 in the Protein Data Bank http://www.rcsb.org/pdb).

With the structure of $\mathrm{C} 3 \mathrm{c}$ available, we were able to solve the structure of $\mathrm{C} 3$ more readily. To retain haemolytic activity C3 was precipitated by dialysis against $5 \mathrm{mM}$ of MES buffer at a $\mathrm{pH}$ of 6.0 and the precipitate was stored at $-80^{\circ} \mathrm{C}$. Shortly before the crystallization experiments precipitated C3 was resolubilized by dialysis against $10 \mathrm{mM}$ of Tris buffer at a $\mathrm{pH}$ of 7.4. In this way, we obtained crystals of native, glycosylated human C3 with the thioester intact. Diffraction data was collected up to 3.3-Å resolution. The structure was solved by molecular replacement using the known structures of C3c and C3d (Nagar et al., 1998) followed by model building to complete the structure. The final model of $\mathrm{C} 3$ consisted of a total of 1611 residues forming 13 domains (entry 2A73 in the Protein Data Bank).

\section{Domain organization}

The structures of C3c and C3 display an intricate arrangement of 10 and 13 domains, respectively (Fig. 1). The core of the protein is formed by eight homologous domains, which we named macroglobulin (MG) domains referring to the related immunoglobulin fold and to the family of $\alpha 2 \mathrm{M}$ proteins. The first five domains, MG1-5, are formed by residues of the $\beta$ chain. Most surprisingly, the next domain, MG6, is formed by residues from both the $\beta$ - and $\alpha$-chains, such that the two chains are intertwined. The last two MG domains, MG7-8, are provided by the $\alpha$-chain. The other five domains are crafted onto this core of eight MG domains in two large insertions and one extension.

The first insert is located in MG6 and is formed by residues 578-745. This segment includes the linker region (LNK), the tetra-arginine pro-C3 processing site, the anaphylatoxin (ANA) domain and a linker ( $\alpha$ 'NT) that connects the ANA domain back to MG6. A remarkable feature of this insert is its geometric arrangement. The insert loops completely through the central hole formed by the MG1-6 domains. Because the processing site is included in this segment, the mature protein appears as two chains that are intertwined in domain MG6. In the structure of $\mathrm{C} 3$, the $\mathrm{C}$-terminus of the $\beta$-chain and $\mathrm{N}$-terminus of the $\alpha$-chain 

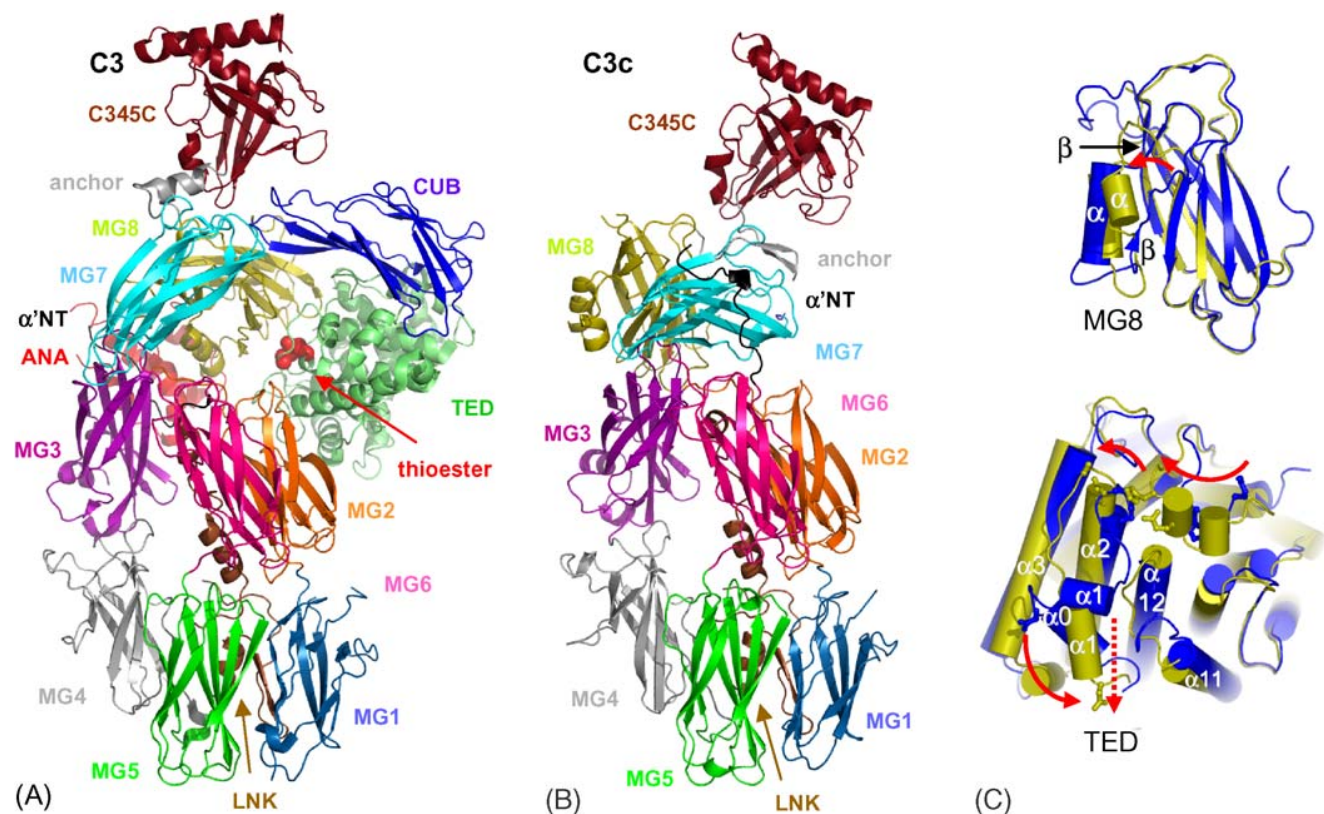

(C)

Fig. 1. Structures of human complement component C3 and C3c. (A) Ribbon representation of C3. Each of its 13 domains is shown in a different colour with domain names as indicated. Additional features indicated are the intact thioester displayed as red spheres, the anchor region shown in grey, and the $\alpha$ 'NT in black. (B) Ribbon representation of C3c, its 10 domains are coloured according to A. (C) Superposed MG8 of C3 (blue) and MG8 of C3c (olive) with the secondary structure elements of the $\beta-\alpha-\beta / \beta-\alpha-\alpha$ transition indicated and superposed TED domain of C3 (blue) and C3d (olive) (Nagar et al., 1998) with residues of the thioester and acyl-imidazole intermediate shown in stick representation. Conformational changes are indicated by red arrows.

are far apart; there is a gap of $53 \AA$ between the last $\beta$ residue (Pro-643) and first $\alpha$ residue (Val-651) present in the model. In pro-C 3 the two ends of the chains are connected through the tetra-arginine (residues 646-649). Thus, in pro-C3 the LNKANA arrangement must be drastically different.

Residues 913-1334 form a segment that may be considered an insert between domains MG7 and MG8. These residues form the CUB domain and the TED domain that carries the reactive thioester. The TED domain itself (residues 963-1268) is inserted in loop $\beta 5-\beta 6$ of the CUB domain. Consequently, CUB is an intertwined domain consisting of two distinct parts of the polypeptide chain (residues 912-962 and 1269-1330). The cleavage fragments $\mathrm{C} 3 \mathrm{~d}, \mathrm{C} 3 \mathrm{~g}$ and $\mathrm{C} 3 \mathrm{f}$ are part of this CUB-TED insert.

At the C-terminal end the $\mathrm{C} 345 \mathrm{C}$ domain is connected to MG8 via a short anchor region. This C-terminal extension is common to the complement proteins $\mathrm{C} 3, \mathrm{C} 4$ and $\mathrm{C} 5$, and not to other members of the $\alpha 2 \mathrm{M}$ family. The anchor region links the C345C domain to both the MG8 domain, through the polypeptide chain, and to the MG7 domain, through a disulphide bridge. As such, the position and orientation of $\mathrm{C} 345 \mathrm{C}$ depends on both MG7 and MG8 by a riding motion.

Overall, the structures of $\mathrm{C} 3$ and $\mathrm{C} 3 \mathrm{c}$ have revealed an intricate arrangement and composition of 13 and 10 domains, respectively (Fig. 1). The data shows that the previously listed A2M_N and $\mathrm{A} 2 \mathrm{M}$ regions consist of the eight $\mathrm{MG}$ domains forming the core and the LNK, CUB and TED domains. Neither sequence alignments nor intron/exon boundaries indicated the observed domains. The fact that intron/exon boundaries do not overlap with domain boundaries is also observed for other ancient multidomain proteins unique to metazoans. This probably results from a continual insertion and removal of introns (Patthy, 1999). This notion of ancientness is further supported by the lack of sequence identity among the eight MG domains, which do not show a single conserved amino-acid residue.

\section{Structural rigidity and flexibility}

Comparison of the structures of $\mathrm{C} 3$ and $\mathrm{C} 3 \mathrm{c}$ suggests that domains MG1-6 form a structurally stable platform, that we called the $\beta$-ring, onto which the flexible domains of the $\alpha$ chain are crafted. Even though large movements of domains occur (with maximum displacements up to $50 \AA$ for residues of MG8) most domains themselves remain relatively unchanged. Three significant exceptions are the MG8 domain, the small anchor region that links C345C to MG7 and MG8; and, the TED domain when compared to the structure of C3d. Another striking structural change involves the linker between the ANA and MG6 domain, referred to as $\alpha^{\prime} \mathrm{NT}$, that will be discussed in Section 5.1.

\subsection{Flexible motif in $M G 8$}

MG8 of C3 plays a crucial role in the double protection mechanism of the highly reactive thioester. First, it contributes a large part of the hydrophobic/aromatic pocket that protects the thioester against nucleophiles; and second, it prevents the formation of the free thiolate and acyl-imidazole intermediate necessary for the high-reactivity with hydroxyl nucleophiles (Law and Dodds, 1997). MG8 drastically changes position from $\mathrm{C} 3$ to $\mathrm{C} 3 \mathrm{c}$. In addition, the domain itself undergoes a conformational change (as shown in Fig. 1C): residues 1388-1420 change 
conformation from a $\beta-\alpha-\beta$ to a $\beta-\alpha-\alpha$ motif in the conversion of $\mathrm{C} 3$ to $\mathrm{C} 3 \mathrm{c}$. This structurally variable segment is not part of the MG core fold (it forms a segment in between strands $\beta C$ and $\beta C$ ' of the MG fold). The $\beta-\alpha-\beta$ motif is also observed in the structure of the isolated RBD of $\alpha 2 \mathrm{M}$. In $\alpha 2 \mathrm{M}$ this segment is important for the binding to the $\alpha 2 \mathrm{M}$ receptor (Jenner et al., 1998). In C3 this segment is involved in protecting the thioester, as it bridges contacts between TED (through Arg-1405, Ser-1408, Lys-1409 and Glu-1411) and the ANA domain (through Gln-1398, Leu1399, Val-1403, Asp-1404 and Tyr-1406) stabilizing the overall structure of C3. In addition, residues 1402-1435 were found to be important for properdin binding (Daoudaki et al., 1988), which stabilizes the $\mathrm{C} 3 \mathrm{bBb}, \mathrm{C} 3$-convertase complex. In contrast to $\mathrm{C} 3$ where the segment is largely buried, the segment is exposed in $\mathrm{C} 3 \mathrm{c}$. We hypothesize that $\mathrm{C} 3 \mathrm{c}$ resembles $\mathrm{C} 3 \mathrm{~b}$ in this respect. The change from hidden to exposed and the conformational change from $\beta-\alpha-\beta$ to a $\beta-\alpha-\alpha$ may be a critical feature for properdin binding to the $\mathrm{C} 3 \mathrm{bBb}$ complex.

\subsection{The anchor region is structurally variable}

The anchor region (residues 1475-1495) connects the Cterminal C345C domain to the body of the molecule via the peptide chain to MG8 and through a disulphide bond (Cys-851 to Cys-1491) to MG7. The anchor region has an additional internal disulphide bond from Cys-1484 to Cys-1489. Surprisingly this region undergoes a drastic conformational change in the conversion of $\mathrm{C} 3$ to $\mathrm{C} 3 \mathrm{c}$ from an $\alpha$-helix to a $\beta$-hairpin, respectively, apparently not restricted by the internal disulphide bond. Possibly the conformational change is induced by the large movement of MG7 in the conversion of $\mathrm{C} 3$ to $\mathrm{C} 3 \mathrm{c}$ onto which the anchor seems to "ride". The conformational change of the anchor might be important for factor B binding (Kolln et al., 2004) in the formation of the $\mathrm{C} 3$ convertase.

\subsection{Conformational changes in the TED domain}

The TED domain forms a double, $\alpha 6-\alpha 6$, helical bundle. The helices alternate outside-in with the even helices forming the central 6 helical bundle and the odd numbered helices forming a shell around the inner bundle. Structures of isolated C3d (Nagar et al., 1998; Zanotti et al., 2000), C4d (van den Elsen et al., 2002) and C3d in complex with CCP domains 1 and 2 of CR2 (Szakonyi et al., 2001) have been solved previously. These structures showed little structural differences among each other. In contrast, large structural changes are apparent between the TED domain of $\mathrm{C} 3$ and the $\mathrm{C} 3 \mathrm{~d}$ structure, which appear correlated with the switch from the thioester to the free thiolate and acyl-imidazole intermediate (Fig. 1C). In $\mathrm{C} 3$ the intact thioester sits on top of helix $\alpha 2$, which adopts a strained $3_{10}$ helical conformation. In C3d the thioester residues move upward (by $3 / 4$ of helical turn) and the helix straightens into a typical $\alpha$-helix conformation. This up, and sideways, movement of helix $\alpha 2$ creates space for loop 1103-1115, which is now free to move in. This loop, which was largely disordered in C3, adopts a welldefined conformation in $\mathrm{C} 3 \mathrm{~d}$, where it forms two short helical turns (1105-1108 and 1110-1112). The straightening of the helix $\alpha 2$ and the upwards movement of the thioester are possibly correlated with changes in the $\mathrm{N}$-terminus of TED. This $\mathrm{N}$-terminus displays two small helices, $\alpha 0$ and $\alpha 1$ that are packed in between helix $\alpha 3$, loop $\alpha 11-\alpha 12$ and helix $\alpha 2$. This region (residues 965981) is extended in C $3 \mathrm{~d}$ with helix $\alpha 0$ expelled. Potentially, these changes may be induced by a pulling force on the $\mathrm{N}$-terminus in the conformational change from $\mathrm{C} 3$ to $\mathrm{C} 3 \mathrm{~b}$.

The remainder of the domain is conformationally stable. By and large, the structural changes are limited to helices $\alpha 0, \alpha 1, \alpha 2$ and $\alpha 3$ that rearrange as discussed. Most of the interaction faces, therefore, do not undergo large changes in themselves and appear structurally stable. In contrast large changes in accessibility are expected for the interfaces of TED in the conversion of C3 to $\mathrm{C} 3 \mathrm{~b}$, i.e. TED is nestled in $\mathrm{C} 3$, whereas a more exposed position is expected for TED in C3b.

\section{Location of binding sites}

$\mathrm{C} 3$ and its derived products are versatile molecules that are able to interact with over 30 different ligands from self and nonself (Lambris, 1988; Lambris et al., 1998); and, new ligands are still being found (Helmy et al., 2006). The affinity for many of these ligands changes in going from $\mathrm{C} 3$ to $\mathrm{C} 3 \mathrm{~b}, \mathrm{iC} 3 \mathrm{~b}$ and finally to $\mathrm{C} 3 \mathrm{c}$ and $\mathrm{C} 3 \mathrm{~d}$ with most ligands binding to the activated forms, $\mathrm{C} 3 \mathrm{~b}$ and $\mathrm{iC} 3 \mathrm{~b}$. Numerous studies have provided insights into the binding sites of these ligands. However, up till now the structural data was lacking to provide a comprehensive view of these binding sites. Here, we discuss the proposed binding sites with regard to the structures of $\mathrm{C} 3$ and $\mathrm{C} 3 \mathrm{c}$ (see also (Janssen and Gros, 2006)) (Fig. 2). Unfortunately, no structural data are yet available for $\mathrm{C} 3 \mathrm{~b}$ and $\mathrm{iC} 3 \mathrm{~b}$. Therefore, potential conformational changes must be considered that will affect the arrangement of the putative binding sites.

\subsection{Convertase formation}

The complement amplification step is initiated by the formation of the $\mathrm{C} 3$ convertase through the binding of factor $\mathrm{B}$ to $\mathrm{C} 3 \mathrm{~b}$ and subsequent proteolytic activation of factor $\mathrm{B}$. By binding to $\mathrm{C} 3 \mathrm{~b}$, factor $\mathrm{B}$ becomes sensitive to factor $\mathrm{D}$ which cleaves it into $\mathrm{Ba}$, which dissociates from the convertase, and $\mathrm{Bb}$, which stays attached. This yields the active (and short-lived) convertase complex of the alternative pathway $\mathrm{C} 3 \mathrm{bBb}$ that will amplify the complement response by cleaving additional $\mathrm{C} 3$ into $\mathrm{C} 3 \mathrm{a}$ and $\mathrm{C} 3 \mathrm{~b}$. So far four separate sites on C $3 \mathrm{~b}$ have been determined that may either represent a binding site or residues that are indirectly involved in the binding of factor $\mathrm{B}$. These putative binding sites are; (i) residues 727-745 (Fishelson, 1991), which form the $\alpha^{\prime} \mathrm{N}$ terminus ( $\alpha$ 'NT), especially acidic residues Asp-730, Glu-731, Glu-736 and Glu-737 (Taniguchi-Sidle and Isenman, 1994); (ii) residues 933-942 (O'Keefe et al., 1988), which form strand $\beta 4$ and its flanking loops in the CUB domain of $\mathrm{C} 3$; (iii) residues 200-220 (Inal and Schifferli, 2002), which form strand $\beta G$ of MG2, the connecting MG2-MG3 loop and $\beta A$ of MG3; and (iv) the C-terminal C345C domain (formed by residues 14961641) (Kolln et al., 2005; Kolln et al., 2004). Together these data indicate four structurally distinct regions located in the top part, 


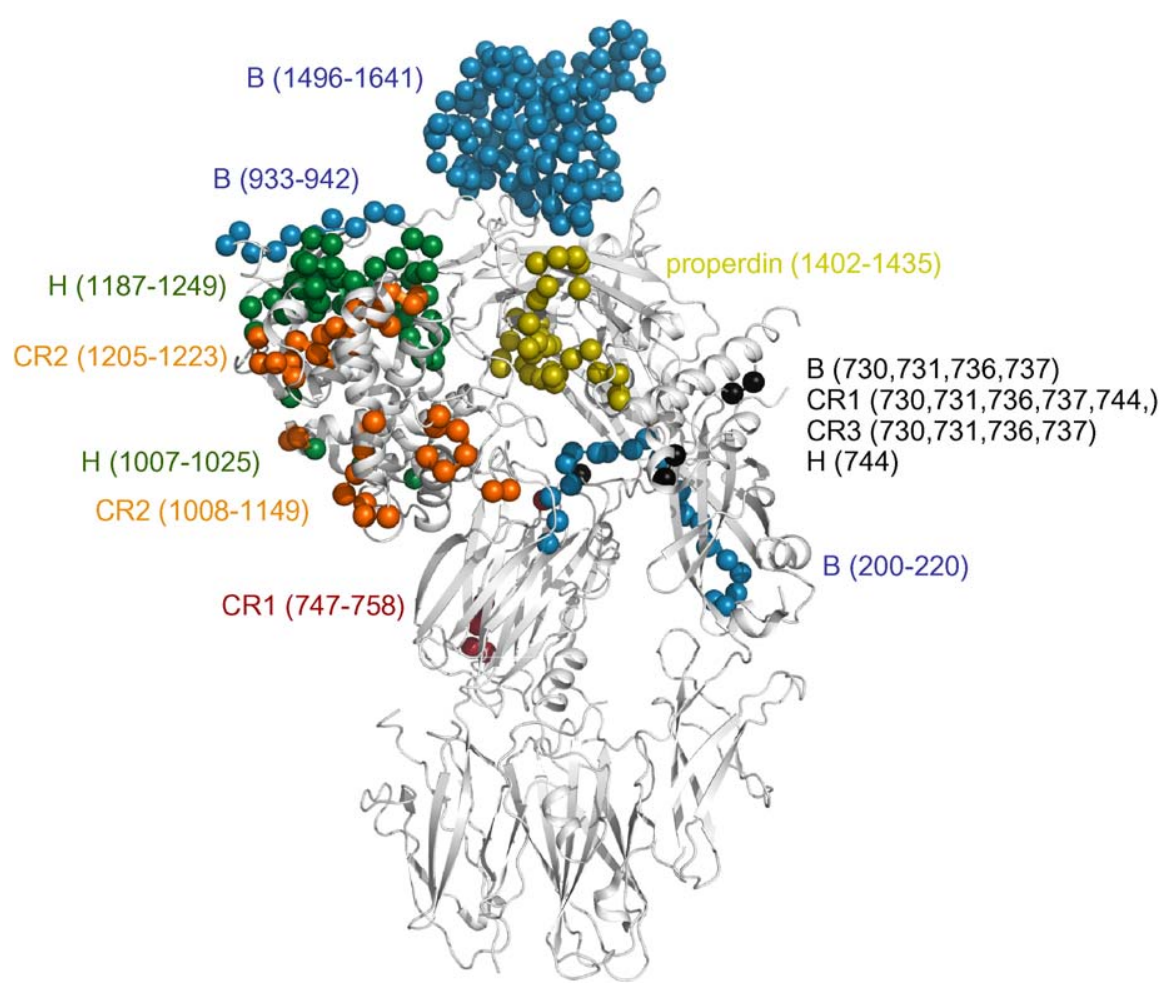

Fig. 2. Putative binding sites mapped on the structure of C3. Proposed binding sites (as discussed in Section 5) are indicated by residue numbering and spheres, coloured according to ligand; factor B (blue), factor H (green), CR1 (red), CR2 (orange), properdin (olive) and the $\alpha$ 'NT containing binding sites for factor B, CR1, $\mathrm{CR} 3$ and factor $\mathrm{H}$ (black).

mostly within the $\alpha$-chain, of the C3 molecule (Fig. 2). Structural changes are expected in the conversion of $\mathrm{C} 3$ to $\mathrm{C} 3 \mathrm{~b}$ (Isenman et al., 1981). Nevertheless the C345C domain is exposed in both $\mathrm{C} 3$ and $\mathrm{C} 3 \mathrm{c}$, which suggests that it is also exposed in $\mathrm{C} 3 \mathrm{~b}$ and thus accessible for factor B binding. The segment formed by residues 933-942 (O'Keefe et al., 1988) on CUB is exposed in C3 and proteolytically removed by factor I in C3c (Lachmann et al., 1982). In the structure of $\mathrm{C} 3$ it is located close to the C345C domain and possibly even after conformational changes factor B can bind simultaneously to both regions. In contrast, residues 200-220 are inaccessible to proteins in both C3 and $\mathrm{C} 3 \mathrm{c}$, even though these residues are exposed to the solvent. A conformational change would be required for factor B binding to this segment. Such a change is not observed in this region when comparing C3 and C3c. Strikingly, the $\alpha$ 'NT region with the four acidic residues required for factor B binding (TaniguchiSidle and Isenman, 1994) is buried in C3 by the ANA domain and surface exposed in $\mathrm{C} 3 \mathrm{c}$. The $\alpha$ 'NT region undergoes a drastic conformational change from $\mathrm{C} 3$ to $\mathrm{C} 3 \mathrm{c}$. By sliding through the ring formed by the $\beta$-chain, it moves from the ANA side to the opposite, MG6 side of the molecule. However, at this stage we do not know in which conversion step this large structural rearrangement of the $\alpha$ 'NT occurs and, thus, on which side the $\alpha$ 'NT resides when factor $\mathrm{B}$ binds to $\mathrm{C} 3 \mathrm{~b}$.

The labile complexes $\mathrm{C} 3 \mathrm{bB}$ and $\mathrm{C} 3 \mathrm{bBb}$ are stabilized over 10-fold by properdin binding (Fearon and Austen, 1975; Hourcade, 2006). Properdin binding increases the affinity of factor $\mathrm{B}$ for $\mathrm{C} 3 \mathrm{~b}$ and prevents the cleavage of $\mathrm{C} 3 \mathrm{~b}$ to $\mathrm{iC} 3 \mathrm{~b}$ by factor $\mathrm{I}$. Residues 1402-1435 form a binding site for properdin (Daoudaki et al., 1988) (Fig. 2). These residues are part of MG8 forming the conformationally flexible $\beta-\alpha-\beta / \beta-\alpha-\alpha$ motif (residues 1388-1420), as discussed above. Not only does this segment alter its conformation, it also changes from hidden to exposed in the conversion of $\mathrm{C} 3$ to $\mathrm{C} 3 \mathrm{c}$. Possibly this site is also exposed in the $\mathrm{C} 3 \mathrm{bB}$ and $\mathrm{C} 3 \mathrm{bBb}$ complexes. Furthermore, the properdin $\mathrm{C} 3 \mathrm{bBb}$ interaction has been proposed to be mainly ionic strength dependent (DiScipio, 1981). This is in good agreement with the mainly positively charged properdin (Sun et al., 2004) and mainly negatively charged C3 and C3c (Janssen et al., 2005).

\subsection{Decay acceleration activity}

Regulation of complement is crucial to prevent severe damage to host tissues. Inhibition of convertase activity, and thus inhibition of complement amplification, is achieved by various regulators that accelerate the dissociation of the convertases, called "decay-acceleration activity" (DAA) (Kirkitadze and Barlow, 2001) and that can discriminate between self and non-self. These regulators belong to a family of homologous proteins called "regulators of complement activation" (RCA). Factor H, decay-accelerating factor (DAF, CD55) and complement receptor 1 (CR1, CD35) are three important members of this family. Various studies (Becherer et al., 1992; Fishelson, 1991; Lambris et al., 1996; Oran and Isenman, 1999; TaniguchiSidle and Isenman, 1994) have shown that residues 727-767 of $\mathrm{C} 3 \mathrm{~b}$ form an important interaction site for both factor $\mathrm{H}$ and CR1. Mutational studies have further pinpointed crucial residues in this region. For factor $\mathrm{H}$ these are acidic residues Glu-744 and 
Glu-747 and for CR1 these are acidic residues Asp-730, Glu731, Glu-736, Glu-737, Glu-747, Glu-754 and Glu-755 located on $\alpha^{\prime}$ NT (729-745) and on MG6 (535-577 and 746-806) (Fig. 2). This indicates that both factor $\mathrm{H}$ and CR1 have a primary binding site on MG6. The fore-mentioned residues are the same or are close to residues important for factor B interaction. This is consistent with the observation that factor $\mathrm{H}, \mathrm{CR} 1$ and factor B compete for $\mathrm{C} 3 \mathrm{~b}$ or $\mathrm{C} 3\left(\mathrm{H}_{2} \mathrm{O}\right)$ binding (Weiler et al., 1976). Factor $\mathrm{H}$ has at least one additional binding site situated on the TED domain (Lambris et al., 1988). Very recently two different models of a complex between $\mathrm{C} 3 \mathrm{~d}$ and the $\mathrm{C}$-terminal two CCP domains (CCP19-20) of factor $\mathrm{H}$ were proposed (Herbert et al., 2006; Jokiranta et al., 2006). One model suggests that residues Asp-1007, Glu-1008, Glu-1010, Arg-1020, Glu-1025 and Lys-1262 of TED/C3d are involved in factor $\mathrm{H}$ binding (Jokiranta et al., 2006), whereas the other proposes a binding site somewhere between residues 1187-1249 (Herbert et al., 2006) (Fig. 2). Residues proposed in both cases are for a large part solvent exposed in C3, however some parts of the 1187-1249 region are hidden, i.e. beginning of helix $\alpha 10$ (by MG8), a side of $\alpha 11$ (by CUB), loop $\alpha 11-\alpha 12$ (by CUB) and the N-terminal part of $\alpha 12$ (by MG8), which could explain the specificity of factor $H$ for $\mathrm{C} 3 \mathrm{~b}$ and not $\mathrm{C} 3$. However, factor $\mathrm{H}$ is an extended molecule consisting of $20 \mathrm{CCP}$ domains, steric hindrance of this or other interaction sites determining specificity cannot be excluded at this stage. Thus, the exact mode of interactions involved in DAA remains unclear, but a general model of steric hindrance disrupting the $\mathrm{C} 3 \mathrm{bBb}$ interactions appears plausible.

\subsection{Cofactor activity}

A second mechanism of complement regulation is the processing of $\mathrm{C} 3 \mathrm{~b}$ into $\mathrm{iC} 3 \mathrm{~b}$ by the protease factor I with the aid of additional cofactors. Processing into $\mathrm{iC} 3 \mathrm{~b}$ induces structural rearrangements and changes the binding properties, e.g. factor $\mathrm{B}$ and properdin can no longer bind. The cofactors which display the "cofactor activity" (CA) are factor H, CR1 and membranecofactor protein (MCP) which also belongs to the RCA family of proteins (Medicus et al., 1983; Pangburn et al., 1977; Ross et al., 1982; Seya et al., 1986). This cofactor-induced processing of $\mathrm{C} 3 \mathrm{~b}$ occurs in three cleavage steps. First factor I cleaves between residues Arg-1281 and Ser-1282 in loop $\beta 6-\beta 7$ of the CUB $^{f}$ part of CUB which generates iC $3 b_{1}$. A second cleavage occurs between Arg-1298 and Ser-1299, in strand $\beta 8$ of CUB ${ }^{\mathrm{f}}$ generating C3f (2 kDa) and iC3b 2 (Davis and Harrison, 1982; Harrison and Lachmann, 1980). Third, factor I cleaves between Arg-932 and Ser-933 in loop $\beta 3$ ' $-\beta 4$ of the $\mathrm{CUB}^{\mathrm{g}}$ part, resulting in the formation of C3dg (40 kDa) and C3c (135 kDa) (Lachmann et al., 1982). This indicates that factor I might bind in various ways to the CUB domain assisted by one of the cofactors which might be involved in the unraveling of the CUB domain enabling factor I to cleave.

\subsection{C3b fragments signaling}

Two fragments generated by the processing of C3b, i.e. iC $3 b$ and finally $\mathrm{C} 3 \mathrm{dg}$, have an important role in the initiation of adap- tive immune responses (Carroll, 2004). In addition, iC3b acts as an opsonin resulting in phagocytosis of tagged particles by leukocytes. The recognition of $\mathrm{iC} 3 \mathrm{~b}$ is mediated by the integrin, complement receptor $3\left(\mathrm{CR} 3, \alpha_{\mathrm{M}} \beta_{2}, \mathrm{CD} 11 \mathrm{~b} / \mathrm{CD} 18\right.$, Mac-1) on leukocytes (Kamata et al., 1995; McGuire and Bajt, 1995; Ueda et al., 1994) and by the very recently discovered complement receptor of the immunoglobulin superfamily (CRIg) on Kupffer cells (Helmy et al., 2006). As for factor B, factor $\mathrm{H}$ and CR1 the $\alpha$ 'NT has also been shown to be important for the CR3 interaction (Taniguchi-Sidle and Isenman, 1994), implicating that this region is also exposed in iC3b (Fig. 2). As CR3 does not bind to $\mathrm{C} 3 \mathrm{~b}$ it is possible that there are differences in or near the $\alpha$ 'NT between C3b and iC $3 b$ which determines the specificity. Another possibility is that these ligands bind multiple regions which are conformationally different between $\mathrm{C} 3 \mathrm{~b}$ and $\mathrm{iC} 3 \mathrm{~b}$. This is supported by various studies which show that multiple regions of CR3 contribute to $\mathrm{iC} 3 \mathrm{~b}$ interaction ( $\mathrm{Li}$ and Zhang, 2003; Xiong and Zhang, 2001).

Binding of complement receptor 2 (CR2, CD21), consisting of 15 or $16 \mathrm{CCP}$ domains, with surface bound $\mathrm{iC} 3 \mathrm{~b}$ or $\mathrm{C} 3 \mathrm{dg}$ and the B-cell antigen receptor complex results in the stimulation of B-cells. This provides a link between the innate and the adaptive immune system (Carroll, 2004). Multiple studies have been performed on the interaction of CR 2 with $\mathrm{iC} 3 \mathrm{~b}$ and $\mathrm{C} 3 \mathrm{dg}$ (Clemenza and Isenman, 2000; Diefenbach and Isenman, 1995; Esparza et al., 1991; Kalli et al., 1991; Lambris et al., 1985; Lowell et al., 1989; Molina et al., 1995; Morikis and Lambris, 2004; Sarrias et al., 2001) (Fig. 2). A few years ago, the structure of a complex between C3d and two domains of CR2 (CCP1-2) was solved (Szakonyi et al., 2001). However this complex is controversial. In this complex the two CCP modules of CR2 adopt a V-shaped arrangement that would not be likely for the physiologically glycosylated CR2, which adopts a more extended conformation (Prota et al., 2002). Moreover, this structure of the complex showed interactions only between CCP2 of CR2 and C $3 \mathrm{~d}$ and no direct interactions between CCP1 of CR2 and C3d. Two recent studies by the same authors showed that CCP1 of CR2 probably contacts C3d directly (Gilbert et al., 2005; Hannan et al., 2005). Although significant structural differences are apparent between TED of C3 and C3d, the CR2 CCP2-binding site is very similar in the two structures. This indicates that conformational changes do not play a role for this subsite. Furthermore, this site is completely exposed in native $\mathrm{C} 3$, which would suggest that $\mathrm{CCP} 1$, and not CCP2, of CR2 discriminates between the conformational states of $\mathrm{C} 3$ versus $\mathrm{iC} 3 \mathrm{~b}$ and $\mathrm{C} 3 \mathrm{dg}$. Possibly its binding site is either inaccessible in $\mathrm{C} 3$ or is different in conformation.

\section{Concluding remarks}

The structures of $\mathrm{C} 3$ and $\mathrm{C} 3 \mathrm{c}$ provide a great wealth of information. They show in detail the composition of the molecules and the location of the many interaction sites that are known for $\mathrm{C} 3$ and its activated fragments. However, a number of important questions remain, such as what is the arrangement of domains and, thus, the interaction sites in the activated molecules $\mathrm{C} 3 \mathrm{~b}$ and $\mathrm{iC} 3 \mathrm{~b}$. Furthermore, the mechanisms of convertase formation and regulation are still poorly understood. The structures of $\mathrm{C} 3$ 
and $\mathrm{C} 3 \mathrm{c}$, however, provide a new starting point for additional structural and mutagenesis studies to elucidate the molecular mechanisms underlying the diverse functions of $\mathrm{C} 3$ and its derivatives.

\section{Acknowledgements}

We are grateful to Fin Milder, Michael Hadders and Lucio Gomes (Utrecht) for critically reading the manuscript. We thank the members of the laboratory for Crystal and Structural Chemistry for discussions. Financial support by the Council for Chemical Sciences of the Netherlands Organization of Scientific Research (NWO/CW) is gratefully acknowledged.

\section{References}

Becherer, J.D., Alsenz, J., Esparza, I., Hack, C.E., Lambris, J.D., 1992. Segment spanning residues 727-768 of the complement $\mathrm{C} 3$ sequence contains a neoantigenic site and accommodates the binding of $\mathrm{CR} 1$, factor $\mathrm{H}$, and factor B. Biochemistry 31, 1787-1794.

Blandin, S., Levashina, E.A., 2004. Thioester-containing proteins and insect immunity. Mol. Immunol. 40, 903-908.

Bokisch, V.A., Muller-Eberhard, H.J., Cochrane, C.G., 1969. Isolation of a fragment (C3a). of the third component of human complement containing anaphylatoxin and chemotactic activity and description of an anaphylatoxin inactivator of human serum. J. Exp. Med. 129, 1109-1130.

Bramham, J., Thai, C.T., Soares, D.C., Uhrin, D., Ogata, R.T., Barlow, P.N., 2005. Functional insights from the structure of the multifunctional C345C domain of C5 of complement. J. Biol. Chem. 280, 10636-10645.

Budd, A., Blandin, S., Levashina, E.A., Gibson, T.J., 2004. Bacterial alpha2macroglobulins: colonization factors acquired by horizontal gene transfer from the metazoan genome? Genome Biol. 5, R38.

Carroll, M.C., 2004. The complement system in regulation of adaptive immunity. Nat. Immunol. 5, 981-986.

Chu, C.T., Pizzo, S.V., 1994. alpha 2-Macroglobulin, complement, and biologic defense: antigens, growth factors, microbial proteases, and receptor ligation. Lab. Invest. 71, 792-812.

Clemenza, L., Isenman, D.E., 2000. Structure-guided identification of C3d residues essential for its binding to complement receptor 2 (CD21). J. Immunol. 165, 3839-3848.

Daoudaki, M.E., Becherer, J.D., Lambris, J.D., 1988. A 34-amino acid peptide of the third component of complement mediates properdin binding. J. Immunol. 140, 1577-1580.

Davis III, A.E., Harrison, R.A., 1982. Structural characterization of factor I mediated cleavage of the third component of complement. Biochemistry $21,5745-5749$.

Diefenbach, R.J., Isenman, D.E., 1995. Mutation of residues in the C3dg region of human complement component $\mathrm{C} 3$ corresponding to a proposed binding site for complement receptor type 2 (CR2, CD21) does not abolish binding of iC3b or C3dg to CR2. J. Immunol. 154, 2303-2320.

DiScipio, R.G., 1981. The binding of human complement proteins C5, factor $\mathrm{B}$, beta $1 \mathrm{H}$ and properdin to complement fragment $\mathrm{C} 3 \mathrm{~b}$ on zymosan. Biochem. J. 199, 485-496.

Esparza, I., Becherer, J.D., Alsenz, J., De la Hera, A., Lao, Z., Tsoukas, C.D., Lambris, J.D., 1991. Evidence for multiple sites of interaction in C3 for complement receptor type 2 (C3d/EBV receptor, CD21). Eur. J. Immunol. 21, 2829-2838.

Fearon, D.T., Austen, K.F., 1975. Properd: binding to C3b and stabilization of the C3b-dependent C3 convertase. J. Exp. Med. 142, 856-863.

Fishelson, Z., 1991. Complement C3: a molecular mosaic of binding sites. Mol. Immunol. 28, 545-552.

Gilbert, H.E., Eaton, J.T., Hannan, J.P., Holers, V.M., Perkins, S.J., 2005. Solution structure of the complex between CR2 SCR 1-2 and C3d of human complement: an X-ray scattering and sedimentation modelling study. J. Mol. Biol. 346, 859-873.
Hannan, J.P., Young, K.A., Guthridge, J.M., Asokan, R., Szakonyi, G., Chen, X.S., Holers, V.M., 2005. Mutational analysis of the complement receptor type 2 (CR2/CD21)-C3d interaction reveals a putative charged SCR1 binding site for C3d. J. Mol. Biol. 346, 845-858.

Harrison, R.A., Lachmann, P.J., 1980. Novel cleavage products of the third component of human complement. Mol. Immunol. 17, 219-228.

Helmy, K.Y., Katschke Jr., K.J., Gorgani, N.N., Kljavin, N.M., Elliott, J.M., Diehl, L., Scales, S.J., Ghilardi, N., van Lookeren Campagne, M., 2006. CRIg: a macrophage complement receptor required for phagocytosis of circulating pathogens. Cell 124, 915-927.

Herbert, A.P., Uhrin, D., Lyon, M., Pangburn, M.K., Barlow, P.N., 2006. Disease-associated sequence variations congregate in a polyanionrecognition patch on human factor $\mathrm{H}$ revealed in $3 \mathrm{D}$ structure. $\mathrm{J}$ Biol Chem. 281, 16512-16520.

Hourcade, D.E., 2006. The role of properdin in the assembly of the alternative pathway C3 convertases of complement. J. Biol. Chem. 281, 2128-2132.

Huber, R., Scholze, H., Paques, E.P., Deisenhofer, J., 1980. Crystal structure analysis and molecular model of human $\mathrm{C} 3 \mathrm{a}$ anaphylatoxin. Hoppe Seylers Z. Physiol. Chem. 361, 1389-1399.

Inal, J.M., Schifferli, J.A., 2002. Complement C2 receptor inhibitor trispanning and the beta-chain of $\mathrm{C} 4$ share a binding site for complement $\mathrm{C} 2$. J. Immunol. 168, 5213-5221.

Isenman, D.E., Kells, D.I., Cooper, N.R., Muller-Eberhard, H.J., Pangburn, M.K., 1981. Nucleophilic modification of human complement protein C3: correlation of conformational changes with acquisition of C3b-like functional properties. Biochemistry 20, 4458-4467.

Janssen, B.J., Gros, P., 2006. Conformational Complexity of Complement Component C3. Adv. Exp. Med. Biol. 586, 291-312.

Janssen, B.J., Huizinga, E.G., Raaijmakers, H.C., Roos, A., Daha, M.R., Nilsson-Ekdahl, K., Nilsson, B., Gros, P., 2005. Structures of complement component $\mathrm{C} 3$ provide insights into the function and evolution of immunity. Nature 437, 505-511.

Jenner, L., Husted, L., Thirup, S., Sottrup-Jensen, L., Nyborg, J., 1998. Crystal structure of the receptor-binding domain of alpha 2-macroglobulin. Structure 6, 595-604.

Jokiranta, T.S., Jaakola, V.P., Lehtinen, M.J., Parepalo, M., Meri, S., Goldman, A., 2006. Structure of complement factor H carboxyl-terminus reveals molecular basis of atypical haemolytic uremic syndrome. EMBO. J. 25, 1784-1794.

Kalli, K.R., Ahearn, J.M., Fearon, D.T., 1991. Interaction of iC3b with recombinant isotypic and chimeric forms of CR2. J. Immunol. 147, 590-594.

Kamata, T., Wright, R., Takada, Y., 1995. Critical threonine and aspartic acid residues within the I domains of beta 2 integrins for interactions with intercellular adhesion molecule 1 (ICAM-1) and C3bi. J. Biol. Chem. 270, 12531-12535.

Kirkitadze, M.D., Barlow, P.N., 2001. Structure and flexibility of the multiple domain proteins that regulate complement activation. Immunol. Rev. 180, $146-161$.

Kolln, J., Bredehorst, R., Spillner, E., 2005. Engineering of human complement component $\mathrm{C} 3$ for catalytic inhibition of complement. Immunol. Lett. 98, 49-56.

Kolln, J., Spillner, E., Andra, J., Klensang, K., Bredehorst, R., 2004. Complement inactivation by recombinant human $\mathrm{C} 3$ derivatives. J. Immunol. $173,5540-5545$.

Lachmann, P.J., Pangburn, M.K., Oldroyd, R.G., 1982. Breakdown of C3 after complement activation. Identification of a new fragment $\mathrm{C} 3 \mathrm{~g}$, using monoclonal antibodies. J. Exp. Med. 156, 205-216.

Lagueux, M., Perrodou, E., Levashina, E.A., Capovilla, M., Hoffmann, J.A., 2000. Constitutive expression of a complement-like protein in toll and JAK gain-of-function mutants of Drosophila. Proc. Natl. Acad. Sci. U.S.A. 97, 11427-11432.

Lambris, J.D., 1988. The multifunctional role of C3, the third component of complement. Immunol. Today 9, 387-393.

Lambris, J.D., Avila, D., Becherer, J.D., Muller-Eberhard, H.J., 1988. A discontinuous factor $\mathrm{H}$ binding site in the third component of complement as delineated by synthetic peptides. J. Biol. Chem. 263, 12147-12150.

Lambris, J.D., Ganu, V.S., Hirani, S., Muller-Eberhard, H.J., 1985. Mapping of the $\mathrm{C} 3 \mathrm{~d}$ receptor (CR2)-binding site and a neoantigenic site in the 
C3d domain of the third component of complement. Proc. Natl. Acad. Sci. U.S.A. 82, 4235-4239.

Lambris, J.D., Lao, Z., Oglesby, T.J., Atkinson, J.P., Hack, C.E., Becherer, J.D., 1996. Dissection of CR1, factor H, membrane cofactor protein, and factor B binding and functional sites in the third complement component. J. Immunol. 156, 4821-4832.

Lambris, J.D., Sahu, A., wetsel, R.A., 1998. The chemistry and biology of C3, C4 and C5. In: Volanakis, J.E., Frank, M.M. (Eds.), The Human Complement System in Health and Disease. Marcel Decker, New York, pp. 83-118.

Law, S.K., Dodds, A.W., 1997. The internal thioester and the covalent binding properties of the complement proteins C3 and C4. Protein Sci. 6, 263-274.

Law, S.K., Lichtenberg, N.A., Levine, R.P., 1979. Evidence for an ester linkage between the labile binding site of $\mathrm{C} 3 \mathrm{~b}$ and receptive surfaces. J. Immunol. 123, 1388-1394.

Li, Y., Zhang, L., 2003. The fourth blade within the beta-propeller is involved specifically in C3bi recognition by integrin alpha M beta 2. J. Biol. Chem. 278, 34395-34402.

Lowell, C.A., Klickstein, L.B., Carter, R.H., Mitchell, J.A., Fearon, D.T., Ahearn, J.M., 1989. Mapping of the Epstein-Barr virus and C3dg binding sites to a common domain on complement receptor type 2. J. Exp. Med. 170, 1931-1946.

McGuire, S.L., Bajt, M.L., 1995. Distinct ligand binding sites in the I domain of integrin alpha $\mathrm{M}$ beta 2 that differentially affect a divalent cationdependent conformation. J. Biol. Chem. 270, 25866-25871.

Medicus, R.G., Melamed, J., Arnaout, M.A., 1983. Role of human factor I and $\mathrm{C} 3 \mathrm{~b}$ receptor in the cleavage of surface-bound C3bi molecules. Eur. J. Immunol. 13, 465-470.

Molina, H., Perkins, S.J., Guthridge, J., Gorka, J., Kinoshita, T., Holers, V.M., 1995. Characterization of a complement receptor 2 (CR2, CD21) ligand binding site for $\mathrm{C} 3$. An initial model of ligand interaction with two linked short consensus repeat modules. J. Immunol. 154, 5426-5435.

Morikis, D., Lambris, J.D., 2004. The electrostatic nature of C3d-complement receptor 2 association. J. Immunol. 172, 7537-7547.

Muller-Eberhard, H.J., Gotze, O., 1972. C3 proactivator convertase and its mode of action. J. Exp. Med 135, 1003-1008.

Nagar, B., Jones, R.G., Diefenbach, R.J., Isenman, D.E., Rini, J.M., 1998. $\mathrm{X}$-ray crystal structure of C3d: a C3 fragment and ligand for complement receptor 2. Science 280, 1277-1281.

O'Keefe, M.C., Caporale, L.H., Vogel, C.W., 1988. A novel cleavage product of human complement component $\mathrm{C} 3$ with structural and functional properties of cobra venom factor. J. Biol. Chem. 263, 12690-12697.

Oran, A.E., Isenman, D.E., 1999. Identification of residues within the 727-767 segment of human complement component $\mathrm{C} 3$ important for its interaction with factor $\mathrm{H}$ and with complement receptor 1 (CR1, CD35). J. Biol. Chem. 274, 5120-5130.

Pangburn, M.K., Schreiber, R.D., Muller-Eberhard, H.J., 1977. Human complement C3b inactivator: isolation, characterization, and demonstration of an absolute requirement for the serum protein beta1 $\mathrm{H}$ for cleavage of C3b and C4b in solution. J. Exp. Med. 146, 257-270.
Patthy, L., 1999. Genome evolution and the evolution of exon-shuffling-a review. Gene 238, 103-114.

Prota, A.E., Sage, D.R., Stehle, T., Fingeroth, J.D., 2002. The crystal structure of human CD21: implications for Epstein-Barr virus and C3d binding. Proc. Natl. Acad. Sci. U.S.A. 99, 10641-106416.

Ross, G.D., Lambris, J.D., Cain, J.A., Newman, S.L., 1982. Generation of three different fragments of bound C3 with purified factor I or serum. I. Requirements for factor H vs. CR1 cofactor activity. J. Immunol. 129, 2051-2060.

Sarrias, M.R., Franchini, S., Canziani, G., Argyropoulos, E., Moore, W.T., Sahu, A., Lambris, J.D., 2001. Kinetic analysis of the interactions of complement receptor 2 (CR2, CD21) with its ligands C3d, iC3b, and the EBV glycoprotein gp350/220. J. Immunol. 167, 1490-1499.

Seya, T., Turner, J.R., Atkinson, J.P., 1986. Purification and characterization of a membrane protein (gp45-70) that is a cofactor for cleavage of $\mathrm{C} 3 \mathrm{~b}$ and C4b. J. Exp. Med. 163, 837-855.

Sun, Z., Reid, K.B., Perkins, S.J., 2004. The dimeric and trimeric solution structures of the multidomain complement protein properdin by X-ray scattering, analytical ultracentrifugation and constrained modeling. J. Mol. Biol. 343, 1327-1343.

Sunyer, J.O., Zarkadis, I.K., Lambris, J.D., 1998. Complement diversity: a mechanism for generating immune diversity? Immunol. Today 19, 519-523.

Szakonyi, G., Guthridge, J.M., Li, D., Young, K., Holers, V.M., Chen, X.S., 2001. Structure of complement receptor 2 in complex with its C3d ligand. Science 292, 1725-1728.

Taniguchi-Sidle, A., Isenman, D.E., 1994. Interactions of human complement component $\mathrm{C} 3$ with factor B and with complement receptors type 1 (CR1, $\mathrm{CD} 35)$ and type $3(\mathrm{CR} 3, \mathrm{CD} 11 \mathrm{~b} / \mathrm{CD} 18)$ involve an acidic sequence at the $\mathrm{N}$-terminus of C3 alpha'-chain. J. Immunol. 153, 5285-5302.

Ueda, T., Rieu, P., Brayer, J., Arnaout, M.A., 1994. Identification of the complement iC3b binding site in the beta 2 integrin CR3 (CD11b/CD18). Proc. Natl. Acad. Sci. U.S.A. 91, 10680-10684.

van den Elsen, J.M., Martin, A., Wong, V., Clemenza, L., Rose, D.R., Isenman, D.E., 2002. X-ray crystal structure of the C4d fragment of human complement component C4. J. Mol. Biol. 322, 1103-1115.

Walport, M.J., 2001. Complement. First of two parts. N. Engl. J. Med. 344, 1058-1066.

Weiler, J.M., Daha, M.R., Austen, K.F., Fearon, D.T., 1976. Control of the amplification convertase of complement by the plasma protein beta1H. Proc. Natl. Acad. Sci. U.S.A. 73, 3268-3272.

Xiong, Y.M., Zhang, L., 2001. Structure-function of the putative I-domain within the integrin beta 2 subunit. J. Biol. Chem. 276, 19340-19349.

Zanotti, G., Bassetto, A., Battistutta, R., Folli, C., Arcidiaco, P., Stoppini, M., Berni, R., 2000. Structure at 1.44 A resolution of an N-terminally truncated form of the rat serum complement C3d fragment. Biochim. Biophys. Acta 1478, 232-238.

Zuiderweg, E.R., Nettesheim, D.G., Mollison, K.W., Carter, G.W., 1989. Tertiary structure of human complement component C5a in solution from nuclear magnetic resonance data. Biochemistry 28, 172-185. 